\title{
LOCAL STRUCTURE OF HOLOMORPHIC FOLIATION SINGULARITIES
}

\author{
SinAN SERTÖZ
}

\begin{abstract}
The local behaviour of the leaves of a singular holomorphic foliation is analyzed around the singularity. It is shown that certain integers classify the singular foliation germs. Maps from simpler classes to more complicated classes are constructed and it is shown that every foliation can be expressed in terms of simpler ones, under some mild assumptions.
\end{abstract}

\section{Introduction.}

An outstanding problem in the category of singular holomorphic foliations is the notoriously elusive Rationality Conjecture of Baum and Bott as described in [2]. Some special cases of this conjecture are known but the general case is far from being understood. Since the residues involved in the conjecture are obtained by retracting certain homology classes to the singular set it is reasonable to expect that an understanding of the problem should start with an analysis of the geometry of the singular set of the foliation.

In this article we localize our attention to the structure exhibited by the leaves of a singular holomorphic foliation around its singularity. We show that certain integers are relevant in classifying the singularity type of the foliation. We construct maps from simpler classes to more complicated classes and show that under some reasonable assumptions every foliation can be expressed in terms of simpler ones. Combined with the reductions described in [6] this analysis of the singularity should ease the way to an understanding of the mysteries surrounding the Rationality Conjecture.

This work is also related to the linearization problem of complex holomorphic involutive distributions for which the reader can consult [3]. In our analysis the dimension of the singular set of the foliation emerges as a relevant parameter. The reader can also consult [5] for the fundamental theorem that lies behind the foliation phenomena.

\section{Local Analysis.}

We are interested in a singular holomorphic foliation on a smooth complex manifold. In particular we want to understand the structure of this foliation 
around the singular set. To make this more precise we first establish our notation.

We work on a complex manifold $M$ of dimension $n$, with its holomorphic tangent bundle denoted by $T M$. Throughout $\xi$ denotes an integrable full coherent subsheaf of the tangent sheaf of $M$ of fixed rank $k$. For the technical definition of "full" we refer to [2], [1]. Essentially $\xi$ is full if it is equal to the annihilator of its annihilator: Suppose that a vector field $v$ is annihilated by every differential one-form $\omega$ which annihilates every element of $\xi$, then $\xi$ is full if every such field $v$ belongs to $\xi$. Intuitively speaking an integrable coherent sheaf is full if the algebraic singularities of the sheaf correspond to the geometric singularities of the foliation. It can also be observed that $\xi$ is full if $\tau / \xi$ is torsion free, where $\tau$ denotes the tangent sheaf of $M,[4]$.

Let $\theta_{M}$ denote the structure sheaf of $M$, with $\mu_{M}$ denoting the maximal ideal subsheaf of $\theta_{M}$. If $T_{p} M$ denotes the tangent space of $M$ at the point $p \in M$, define a vector subspace of $T_{p} M$ by $\xi_{p} / \xi_{p} \otimes_{\theta_{p}} \mu_{p}$ and denote it by $T_{p}(\xi)$. It is obtained by germs of $\xi_{p}$ evaluated at $p$. Since $\xi$ is of rank $k$ the dimension of $T_{p}(\xi)$ is $k$ at all points where $\xi$ defines a smooth foliation and this dimension drops at singular points of the foliation. Note that this is in contrast to the number of generators required to span $\xi_{p}$, which is an upper semicontinuous function whereas, the dimension of $T_{p}(\xi)$ is a lower semicontinuous function.

Assume that we have chosen a coordinate system $\left(U, x=\left(x_{1}, \ldots, x_{n}\right)\right)$ centered at $p$. Let $s_{1}, \ldots, s_{l} \in \xi_{p}$ generate $\xi$ on $U$, shrinking $U$ if necessary. Each $s_{i}(p)$ can be considered as a column vector of the form

$$
s_{i}(p)=\left(\begin{array}{c}
s_{1 i}(p) \\
\vdots \\
s_{n i}(p)
\end{array}\right)
$$

in the vector space $T_{p} M$, for $i=1, \ldots, l$. The $n \times l$ matrix

$$
G(p)=\left(\begin{array}{ccc}
s_{11}(p) & \ldots & s_{1 l}(p) \\
\vdots \\
s_{n 1}(p) & \ldots & s_{n l}(p)
\end{array}\right)_{n \times l}
$$

has rank $k$ at $p$ if the foliation is smooth at $p$, and the rank drops if $p$ is a singular point for the foliation.

\subsection{The Filtration of the Singular Set.}

We can now describe the singular set $Z$ of the foliation as

$$
Z=\left\{p \in M \mid \operatorname{dim} T_{p}(\xi)<k\right\}
$$


Our analysis being local we assume that $Z$ is irreducible and connected. Using the representation given in Equation (1) we can describe a filtration of the singular set $Z$ as

$$
Z=Z^{k-1} \supset Z^{k-2} \supset \cdots \supset Z^{0}
$$

where for $i=0, \ldots, k-1$ we define $Z^{i}$ as

$$
Z^{i}=\left\{p \in M \mid \operatorname{dim} T_{p}(\xi) \leq i\right\}
$$

Each $Z^{i}$ is defined as the vanishing set of some minors of the matrix $G$, and is a closed subvariety of $M$. In particular if $Z^{i}-Z^{i-1}$ is not empty then, $Z^{i-1}$ being a closed subvariety of $Z^{i}$, every point in $Z^{i}-Z^{i-1}$ contains an open neighbourhood in $M$ disjoint from $Z^{i-1}$.

\subsection{Germs of Singularities.}

$M$ is a complex manifold of dimension $n$ and we are interested in the local behaviour of the given foliation so we might as well be interested in the local behaviour of a foliation defined around the origin in $\mathbb{C}^{n}$ where the singular set contains the origin.

Consider $U$ as an open neighbourhood of the origin in $\mathbb{C}^{n}$ and let $\xi$ be a full coherent integrable subsheaf of the tangent sheaf of $\mathbb{C}^{n}$ over $U$, of fixed rank $k$. Denote the singular set of the foliation by $Z$ and assume that $Z$ contains the origin. We make the following assumptions on $Z$, taking a smaller $U$ if necessary:

$$
Z \text { is smooth and contains the origin }
$$

$$
\begin{aligned}
\operatorname{dim} Z & =r \\
\operatorname{dim} T_{p}(\xi) & =s \text { for all } p \in Z .
\end{aligned}
$$

The last condition amounts to saying that $Z^{s-1}$ is empty. This can always be satisfied by taking a smaller $U$ since $Z^{s-1}$ is a closed subset in $Z^{s}$. Thus given any four integers

$$
n>k>r \geq s \geq 0
$$

we can consider the set of pairs $\langle\xi, U\rangle$ as above. This set is too big. We define an obvious equivalence relation on this set. Two such elements $\left\langle\xi_{1}, U_{1}\right\rangle$ and $\left\langle\xi_{2}, U_{2}\right\rangle$ are defined to be equivalent if there are an open subset $W$ of $U_{1} \cap U_{2}$ and an invertible holomorphic map $f: W \rightarrow W$ such that $f^{*} \xi_{2}\left|W=\xi_{1}\right| W$. The quotient space under this relation is denoted by $\operatorname{Fol}(n, k, r ; s)$.

Definition 1. The quotient space $\operatorname{Fol}(\mathbf{n}, \mathbf{k}, \mathbf{r} ; \mathbf{s})$ consists of the germs of foliations of rank $k$ foliating an open neighbourhood of the origin in $\mathbb{C}^{n}$ with a singular set $Z$ satisfying the conditions (2), (3) and (4). 
We show in Section 4 that most of these germ spaces are not empty. As a notational convention $\xi$ denotes both the sheaf itself and the equivalence class of the pair $\langle\xi, U\rangle$ in $\operatorname{Fol}(n, k, r ; s)$ and the difference is made clear in the context.

\subsection{First Construction.}

If $\xi$ is in $\operatorname{Fol}(n, k, r ; s)$ and $m$ any non-negative integer we construct in a natural way an element $\xi_{m}$ in $\operatorname{Fol}(n+m, k+m, r+m ; s+m)$. We aim eventually to show that every element of $\operatorname{Fol}(n+m, k+m, r+m ; s+m)$ is of the form $\xi_{m}$ for some $\xi$ in $\operatorname{Fol}(n, k, r ; s)$. This is called the first construction of type $m$ for which we give examples in Section 4 .

If $\xi$ is a foliation around the origin in $\mathbb{C}^{n}$ let the sections $s_{1}, \ldots, s_{l}$ generate it on some neighbourhood $U$. On some open neighbourhood $V$ of $\mathbb{C}^{m}$ we take the independent sections $t_{1}, \ldots, t_{m}$ of the tangent space on $V$. Considering $\mathbb{C}^{n+m}$ as $\mathbb{C}^{n} \times \mathbb{C}^{m}$ we have the sections $\left(s_{1}, 0\right), \ldots,\left(s_{l}, 0\right),\left(0, t_{1}\right), \ldots,\left(0, t_{m}\right)$ of the tangent space of $\mathbb{C}^{n+m}$. These sections generate a full coherent subsheaf of rank $k+m$ on $U \times V$. Call this foliation $\xi_{m}$.

Lemma 2. The pair $\left\langle\xi_{m}, U \times V\right\rangle$ defines an equivalence class in $\operatorname{Fol}(n+$ $m, k+m, r+m ; s+m)$.

Proof. The increments in $n, k$ and $r$ being immediate we have to justify only the increment in $s$. The sections $\left(0, t_{1}\right), \ldots,\left(0, t_{m}\right)$ evaluate to independent vectors at every point. In particular if the singular set of $\xi$ is $Z$ in $U$ then the singular set of $\xi_{m}$ is $Z \times V$ in $U \times V$. And for every point $p \in Z$ and $q \in V$ the vector space $T_{(p, q)}\left(\xi_{m}\right)$ has rank $s+m$, where $s$ is contributed by the sections $\left(s_{1}, 0\right), \ldots,\left(s_{l}, 0\right)$ and $m$ is contributed by $\left(0, t_{1}\right), \ldots,\left(0, t_{m}\right)$.

This gives a map from $\operatorname{Fol}(n, k, r ; s)$ into $\operatorname{Fol}(n+m, k+m, r+m ; s+m)$ whose surjectivity is discussed in Section 3.

\subsection{Second Construction.}

If $\xi$ is in $\operatorname{Fol}(n, k, r ; s)$ as in Section 2.3 then we define a simple lifting of $\xi$ to an element $\ell_{m}(\xi)$ in $\operatorname{Fol}(n+m, k, r+m ; s)$. Note how the dimension of the space and the singular set is incremented but the leaf dimension and the rank of the sheaf over the singularity remain the same. This is called the second construction of type $m$.

Using the notation of the previous section let $\xi \in \operatorname{Fol}(n, k, r ; s)$ be generated by its sections $s_{1}, \ldots, s_{l}$ on $U$. Then the sections $\left(s_{1}, 0\right), \ldots,\left(s_{l}, 0\right)$ describe an integrable full subsheaf of the tangent sheaf of $U \times V$, which we denote by $\ell_{m}(\xi)$.

Lemma 3. If $\xi$ is in $\operatorname{Fol}(n, k, r ; s)$ then $\left\langle\ell_{m}(\xi), U \times V\right\rangle$ defines an equivalence class in $\operatorname{Fol}(n+m, k, r+m ; s)$. 
Proof. We have to justify the new numerical invariants. That $\ell_{m}(\xi)$ defines a foliation in $\mathbb{C}^{n+m}$ is clear. The sections generating $\ell_{m}(\xi)$ are the $\left(s_{i}, 0\right)$ 's which have rank $k$ on $(U-Z) \times V$ since the $s_{i}$ 's have rank $k$ on $U-Z$. The singular set of $\ell_{m}(\xi)$ is $Z \times V$. The dimension of $Z \times V$ is $r+m$. And finally the sections $s_{i}$ 's have rank $s$ on $Z$ and hence the sections $\left(s_{i}, 0\right)$ 's have the same rank on $Z \times V$.

We thus have a map $\ell_{m}$ from $\operatorname{Fol}(n, k, r ; s)$ into $\operatorname{Fol}(n+m, k, r+m ; s)$. Not every foliation in $\operatorname{Fol}(n+m, k, r+m ; s+m)$ is of the form $\ell_{m}(\xi)$ for some $\xi$ in $\operatorname{Fol}(n, k, r ; s)$, and those which are of this form have a special geometry which we single out in the following definition and describe in Section 4.

Definition 4. A foliation $\xi$ of the form $\operatorname{Fol}(n, k, r ; 0)$ is called split if it is of the form $\ell_{r}(\alpha)$ for some $\alpha$ in $\operatorname{Fol}(n-r, k, 0 ; 0)$.

\section{The Structure Theorem.}

In this section we prove that every foliation can be obtained through a first construction of type $m$ as described in Section 2.3.

Theorem 5. Every foliation $\xi$ in $\operatorname{Fol}(n, k, r ; s)$ is of the form $\eta_{s}$ for some $\eta$ in $\operatorname{Fol}(n-s, k-s, r-s ; 0)$. In other words every foliation can locally be projected along its leaves down to a foliation whose rank at the singularity is zero.

Proof. Since we have $n>k>r \geq s$ as in Equation (5) the above statement makes sense as far as the indexes are involved. Let $\xi$ be a foliation in $\operatorname{Fol}(n, k, r ; s)$ defined on an open neighbourhood $U$ of the origin in $\mathbb{C}^{n}$. Let $Z$ be the singular set of $\xi$ satisfying the conditions (2), (3) and (4). Choose a coordinate system $(z, U)$ centered at the origin, after shrinking $U$ if necessary, in such a way that

$$
\begin{aligned}
Z & =\left\{p \in U \mid z_{r+1}(p)=\cdots=z_{n}(p)=0\right\} \\
& =\{(\underbrace{*, \ldots, *}_{s}, \underbrace{*, \ldots, *}_{r-s}, \underbrace{0, \ldots, 0}_{n-r}) \in U\} .
\end{aligned}
$$

We decompose $Z$ into two parts as suggested by the above notation;

$$
\begin{aligned}
Z_{\text {front }} & =\left\{p \in Z \mid z_{s+1}(p)=\cdots=z_{r}(p)=0\right\} \\
& =\{(\underbrace{*, \ldots, *}_{s}, \underbrace{0, \ldots, 0}_{r-s}, \underbrace{0, \ldots, 0}_{n-r}) \in U\}
\end{aligned}
$$


and

$$
\begin{aligned}
Z_{\mathrm{back}} & =\left\{p \in Z \mid z_{1}(p)=\cdots=z_{s}(p)=0\right\} \\
& =\{(\underbrace{0, \ldots, 0}_{s}, \underbrace{*, \ldots, *}_{r-s}, \underbrace{0, \ldots, 0}_{n-r}) \in U\} .
\end{aligned}
$$

We also define a disk $D$ in $U$ as

$$
\begin{aligned}
D & =\left\{p \in U \mid z_{1}(p)=\cdots=z_{s}(p)=0\right\} \\
& =\{(\underbrace{0, \ldots, 0}_{s}, \underbrace{*, \ldots, *}_{r-s}, \underbrace{*, \ldots, *}_{n-r}) \in U\} .
\end{aligned}
$$

With these definitions we have the following relations:

$$
\begin{aligned}
\operatorname{dim} Z_{\text {front }} & =s \\
\operatorname{dim} Z_{\text {back }} & =r-s \\
\operatorname{dim} D & =n-s \\
Z & \cong Z_{\text {front }} \times Z_{\text {back }} \\
Z_{\text {back }} & \subset D \\
U & \cong Z_{\text {front }} \times D .
\end{aligned}
$$

The integrable sheaf $\xi$ restricted to $Z$ defines a smooth foliation of rank $s$ on the $r$-dimensional smooth space $Z$, (see the description of $\operatorname{Fol}(n, k, r ; s)$ in Definition 1 together with the Equation (2), (3) and (4)).

By the Frobenius theorem we can choose a coordinate system $\left(x=\left(x_{1}, \ldots\right.\right.$, $\left.x_{r}\right), W$ ) centered at the origin, where $W$ is an open neighbourhood in $Z$, such that the vector fields $\frac{\partial}{\partial x_{1}}, \ldots, \frac{\partial}{\partial x_{s}}$ generate the vector space $T_{p}(\eta)$ at every point $p$ in $Z$, (see for Example [7].) The coordinate functions $z_{1}, \ldots, z_{r}$ also define a coordinate system on $Z$, (as in Equation (6)). We can then talk about a change of coordinates

$$
\begin{aligned}
x_{1} & =\phi_{1}\left(z_{1}, \ldots, z_{r}\right) \\
& \vdots \\
x_{r} & =\phi_{r}\left(z_{1}, \ldots, z_{r}\right) .
\end{aligned}
$$

These $\phi_{i}$ 's are holomorphic functions defined on $Z \cong \mathbb{C}^{r}$, so extend holomorphically to an open subset in $\mathbb{C}^{n}$ containing $W$, which we can still denote by $U$. This has the effect of extending the functions $x_{i}$ 's and hence the vector fields $\frac{\partial}{\partial x_{1}}, \ldots, \frac{\partial}{\partial x_{s}}$ to $U$. Moreover we can take

$$
\left(x_{1}, \ldots, x_{r}, z_{r+1}, \ldots, z_{n}\right): U \longrightarrow \mathbb{C}^{n}
$$


as a new coordinate chart on $U$.

We will continue the proof of Theorem 5 after the following lemma and its corollary. The notation is as in the theorem.

Lemma 6. There exist vector fields $V_{1}, \ldots, V_{s}$ on $U$, (shrinking $U$ if necessary), which take values in $T_{p}(\xi)$ for all $p \in U$ and generating it for all $p \in Z$.

Proof. Let $V_{1}, \ldots, V_{l}$ be sections of the sheaf $\xi$ in some neighbourhood of the origin, generating the vector spaces $T_{p}(\xi)$ for every $p$ in that neighbourhood. Assume that the sections $V_{1}, \ldots, V_{s}$, (after some reordering if necessary), are such that $V_{1}(\underline{\mathbf{o}}), \ldots, V_{s}(\underline{\mathbf{o}})$ are linearly independent in $T_{\underline{\mathbf{o}}}(\xi)$, where $\underline{\mathbf{o}}$ denotes the origin. Then these $V_{i}$ 's remain linearly independent in $T_{p}(\xi)$ for $p$ in some neighbourhood of the origin. If we denote again by $Z$ the part of $Z$ lying in this neighbourhood, then $V_{1}(p), \ldots, V_{s}(p)$ generate $T_{p}(\xi)$ for all $p \in Z$ since the ranks of these vector spaces are all $s$ by the assumption in Equation $(4)$.

Corollary 7. The sheaf $\xi$ has a subsheaf $\alpha$ of rank $s$ whose complement in $\xi$, which we denote by $\eta$, defines on $D$ a foliation of type $\operatorname{Fol}(n-s, k-$ $s, r-s ; 0)$.

Proof. The sections $V_{1}, \ldots, V_{s}$ generate the subsheaf $\alpha$ around the origin. By the assumptions on $Z$ the rank of $\alpha$ is $s$ throughout. For a fixed trivialization around the origin each vector space $T_{p}(\xi)$ is endowed with the standard inner product. Apply the Gram-Schmidt operation to $V_{1}, \ldots, V_{s}$ to obtain 'orthogonal' sections $V_{1}^{\prime}, \ldots, V_{s}^{\prime}$. Then for each $i=s+1, \ldots, l$ define the section $V_{i}^{\prime}$ of $\xi$ such that

$$
V_{i}^{\prime}(p)=V_{i}(p)-\sum_{j=1}^{s} \frac{V_{i}(p) \cdot V_{j}^{\prime}(p)}{V_{j}^{\prime}(p) \cdot V_{j}^{\prime}(p)} \cdot V_{j}^{\prime}(p),
$$

for all $p \in U$. Heuristically speaking the sections $V_{s+1}^{\prime}, \ldots, V_{l}^{\prime}$ are obtained from the sections $V_{s+1}, \ldots, V_{l}$ by removing their components lying in $\alpha$, in a manner made explicit in the above equation. The sections $V_{s+1}^{\prime}, \ldots, V_{l}^{\prime}$ generate a subsheaf $\eta$ of $\xi$ which can be considered as the complement of $\alpha$. At each point $p \in U$ the vector space $T_{p}(\xi)$ is isomorphic by construction to $T_{p}(\alpha) \oplus T_{p}(\eta)$. So the rank of $\eta$ is $k-s$ on $U-Z$ and drops to zero on $Z$. Now restrict $\eta$ to $D$, (see Equation (9) to recall the definition of $D$ ). $D$ is of dimension $n-s$, and the set on $D$ where the rank of $\eta$ falls to zero is $D \cap Z$ which is $Z_{\text {back }}$ whose dimension is $r-s$. This describes the foliation $\eta$ on $D$ of type $\operatorname{Fol}(n-s, k-s, r-s ; 0)$, as claimed. 
We now continue with the proof of Theorem 5:

Proof [cont' $d]$. Each of the $V_{i}(p)$ 's for $p \in U$ is a linear combination of the $\left.\frac{\partial}{\partial x_{i}}\right|_{p}$ 's:

$$
V_{i}(p)=\left.a_{i 1}(p) \frac{\partial}{\partial x_{1}}\right|_{p}+\cdots+\left.a_{i s}(p) \frac{\partial}{\partial x_{s}}\right|_{p}
$$

where $i=1, \ldots, s, p \in U$ and the $a_{i j}$ 's are holomorphic functions. The matrix

$$
A(p)=\left(a_{i j}(p)\right)_{s \times s}
$$

is an invertible $s \times s$ matrix for every $p \in U$ since the $V_{i}$ 's are linearly independent at each point in $U$.

To follow the arguments of the rest of the proof it may be helpful occasionally to consult to Figure 1.

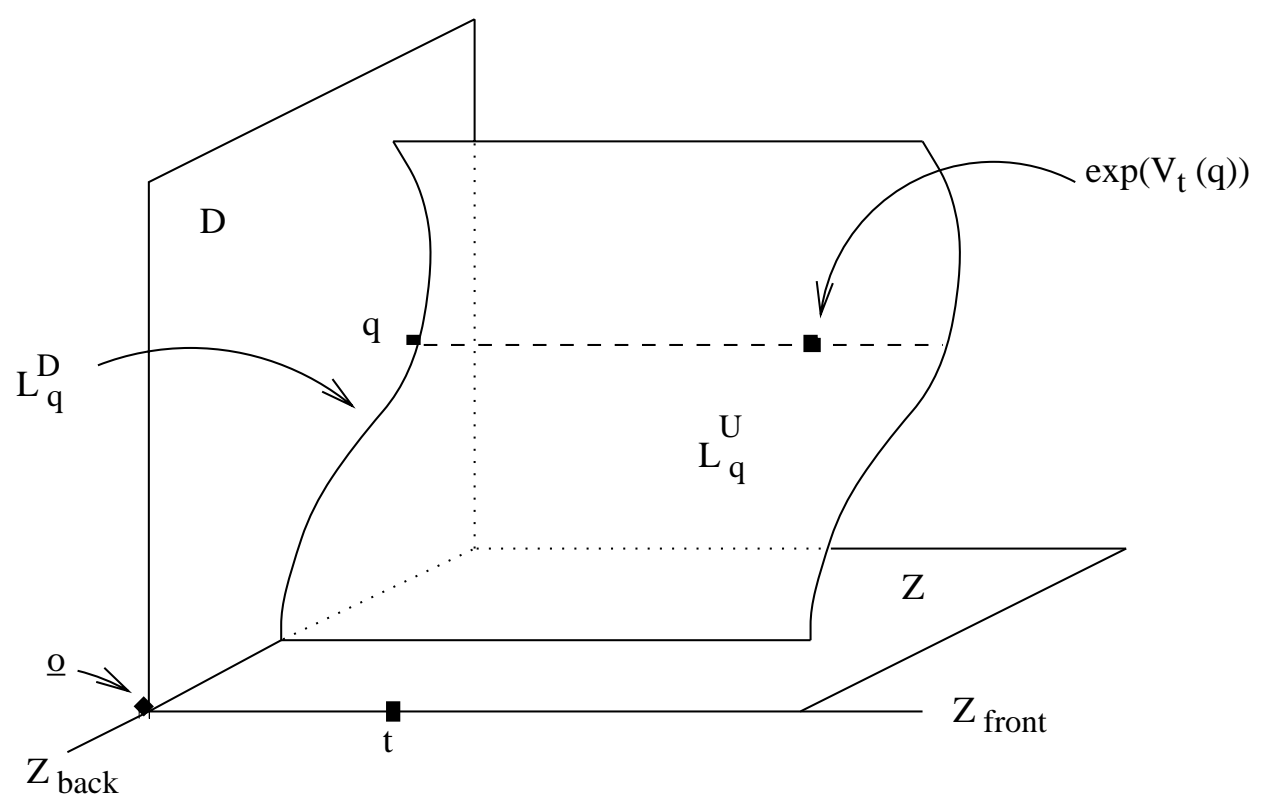

Figure 1. Collapsing $L_{q}^{U}$ down to $L_{q}^{D}$.

For every $t=\left(t_{1}, \ldots, t_{s}, 0, \ldots, 0\right)$ in $Z_{\text {front }}$ define a vector field on $U$ as

$$
V_{t}(p)=t_{1} V_{1}(p)+\cdots+t_{s} V_{s}(p), \quad p \in U .
$$

Recall that

$$
\begin{aligned}
\exp _{V_{t}(p)}: \mathbb{C} & \longrightarrow U \\
\tau & \longmapsto\left(c_{1}(\tau), \ldots, c_{n}(\tau)\right)
\end{aligned}
$$


is the unique map that sends $0 \in \mathbb{C}$ to $p \in U$ and whose differential sends $\left.\frac{d}{d \tau}\right|_{0}$ to $V_{t}(p)$. The usual exponential function is then defined as $\exp \left(V_{t}(p)\right):=\exp _{V_{t}(p)}(1)$. Our plan is to fill out $U$ by copies of $D$ under the exponential map, as we will make explicit below.

Let $q=\left(0, \ldots, 0, q_{s+1}, \ldots, q_{n}\right)$ in $D$ and $t=\left(t_{1}, \ldots, t_{s}, 0, \ldots, 0\right)$ in $Z_{\text {front }}$ be given. For any $1 \leq i_{0} \leq s$ we have

$$
\begin{aligned}
\left(d \exp _{V_{t}(q)}\right)\left(\frac{d}{d \tau}\right)\left(x_{i_{0}}\right) & =\frac{d}{d \tau}\left(x_{i_{0}} \circ \exp _{V_{t}(q)}\right) \\
& =\frac{d}{d \tau} c_{i_{0}}(\tau) .
\end{aligned}
$$

On the other hand the definition gives

$$
\begin{aligned}
\left(d \exp _{V_{t}(q)}\right)\left(\frac{d}{d \tau}\right)\left(x_{i_{0}}\right) & =V_{t}(q)\left(x_{i_{0}}\right) \\
& =\left(t_{1} V_{1}(q)+\cdots+t_{s} V_{s}(q)\right)\left(x_{i_{0}}\right) \\
& =\left(t_{1} \sum_{j=1}^{s} a_{1 j}(q) \frac{\partial}{\partial x_{j}}+\cdots t_{s} \sum_{j=1}^{s} a_{s j}(q) \frac{\partial}{\partial x_{j}}\right)\left(x_{i_{0}}\right) \\
& =t_{1} a_{1 i_{0}}(q)+\cdots+t_{s} a_{s i_{0}}(q) \\
& =A(q)\left(\begin{array}{c}
t_{1} \\
\vdots \\
t_{s}
\end{array}\right) \\
& =\left(\begin{array}{c}
t_{1}^{\prime} \\
\vdots \\
t_{s}^{\prime}
\end{array}\right),
\end{aligned}
$$

where Equation (13) follows from the description of the matrix $A$ in (11), and Equation (14) defines $t_{1}^{\prime}, \ldots, t_{s}^{\prime}$.

If on the other hand $s+1 \leq i_{0} \leq n$, then as above

$$
\left(d \exp _{V_{t}(q)}\right)\left(\frac{d}{d \tau}\right)\left(z_{i_{0}}\right)=\frac{d}{d \tau} c_{i_{0}}(\tau)
$$

and

$$
V_{t}(q)\left(z_{i_{0}}\right)=0
$$

since $V_{t}$ does not contain any $\frac{\partial}{\partial z_{i_{0}}}$. Recalling that $q=\left(0, \ldots, 0, q_{s+1}, \ldots, q_{n}\right)$ the Equations (12) \& (14) and (15) \& (16) give us the following boundary 
value equations;

$$
\begin{aligned}
& \frac{d}{d \tau} c_{i}(\tau)=t_{i}^{\prime}, c_{i}(0)=0, \quad \text { for } 1 \leq i \leq s \\
& \frac{d}{d \tau} c_{i}(\tau)=0, c_{i}(0)=q_{i}, \text { for } \quad s+1 \leq i \leq n
\end{aligned}
$$

Solving these equations we find an explicit expression for the flow;

$$
\begin{aligned}
\exp _{V_{t}(q)}(\tau) & =\left(c_{1}(\tau), \ldots, c_{n}(\tau)\right) \\
& =\left(t_{1}^{\prime} \tau, \ldots, t_{s}^{\prime} \tau, q_{s+1}, \ldots, q_{n}\right) .
\end{aligned}
$$

And finally we have the expression for the exponential map

$$
\exp \left(V_{t}(q)\right)=\left(t_{1}^{\prime}, \ldots, t_{s}^{\prime}, q_{s+1}, \ldots, q_{n}\right)
$$

At this point in the proof we introduce a notation to make the ensuing arguments shorter.

Notation 8. If $M$ is a foliated manifold, singularly or otherwise, and $q$ is a point in $M$ outside the singularity of the foliation, then let $\mathbf{L}_{\mathbf{q}}^{\mathbf{M}}$ denote the connected component of the leaf of the foliation passing through $q$ and contained in a sufficiently small neighbourhood of $q$ which does not intersect the singular set.

Proof [cont' $d]$. We are interested in particular in the leaf germ $L_{q}^{D}$ for the foliation $\eta$ on $D$ and the leaf germ $L_{q}^{U}$ for the foliation $\xi$ on $U$, when $q=$ $\left(0, \ldots, 0, q_{s+1}, \ldots, q_{n}\right)$ is in $D$. Since $T_{q}(\eta)$ is a subsheaf of $T_{q}(\xi)$ we have $L_{q}^{D} \subset L_{q}^{U}$. The vector field $V_{t}(q)$ lies in $T_{p}(\xi)$ for all $p$ in $U$, so the flow generated by $V_{t}(q)$ moves $L_{q}^{D}$ inside $L_{q}^{U}$. Note that

$$
\begin{aligned}
& \operatorname{dim} L_{q}^{D}=k-s \\
& \operatorname{dim} L_{q}^{U}=k .
\end{aligned}
$$

Since $t$ is chosen freely from $Z_{\text {front }}$ whose dimension is $s$, it suffices for our purposes to prove the following equality:

$$
\bigcup_{t \in Z_{\text {front }}} \exp \left(V_{t}\left(L_{q}^{D}\right)\right)=L_{q}^{U}, \quad \text { for all } q \in D
$$

Observe that any point $p$ in $L_{q}^{U}$ is of the form $p=\left(p_{1}, \ldots, p_{s}, q_{s+1}, \ldots, q_{n}\right)$ where $q=\left(0, \ldots, q_{s+1}, \ldots, q_{n}\right) \in D$. To prove the equality (17), and hence to 
complete the proof, we need to produce a point $t=\left(t_{1}, \ldots, t_{s}, 0, \ldots, 0\right) \in Z_{\text {front }}$ which gives $\exp \left(V_{t}(q)\right)=p$. We can choose $t$ as follows

$$
\left(\begin{array}{c}
t_{1} \\
\vdots \\
t_{s}
\end{array}\right)=A^{-1}(q)\left(\begin{array}{c}
p_{1} \\
\vdots \\
p_{s}
\end{array}\right) .
$$

The definition of the matrix in (11) together with the Equations (13) and (14) imply that this choice of $t$ gives $\exp \left(V_{t}(q)\right)=p$, and hence completes the proof. We have shown that every $\xi \in \operatorname{Fol}(n, k, r ; s)$, satisfying the conditions (2), (3) and (4), is of the form $\eta_{s}$ for some $\eta \in \operatorname{Fol}(n-s, k-s, r-s ; 0)$.

At this point we can refer back to Figure 1 for explaining the result in non-technical parlance. Locally, coordinates can be chosen around a singularity such that the leaves can be projected down to a simpler foliation. This projection has the effect of collapsing the leaves to smaller dimensional leaves. The resulting foliation is simpler in the sense that the rank of the vector space obtained by evaluating all the sections of the defining sheaf at the singularity has dimension zero. In Figure 1 the leaf of the foliation is $L_{q}^{U}$. It is collapsed to $L_{q}^{D}$ which is a leaf of the simpler foliation on $D$. Moreover the singular set $Z$ is collapsed down to $Z_{\text {back }}$ which is the singular set of the foliation on $D$.

\section{Examples.}

In this section we construct in detail some examples to show that the germ spaces $\operatorname{Fol}(n, k, r ; s)$, as defined in Section 2.2, are not empty for a large choice of the integers $n, k, r$ and $s$. We will also demonstrate the constructions of the Sections 2.3 and 2.4. In particular we inquire when foliations are split. The first two examples are easy and prepare the setting for the third example.

\section{1. $\operatorname{Fol}(\mathrm{n}, \mathrm{n}-1,0 ; 0)$.}

The simplest singular foliation can be described in $\mathbb{C}^{n}$ by hypersurfaces with a singularity at the origin. To describe this foliation define for any $\lambda \in \mathbb{C}$

$$
L_{\lambda}=\left\{\left(X_{1}, \ldots, X_{n}\right) \in \mathbb{C}^{n} \mid X_{1}^{2}+\cdots+X_{n}^{2}=\lambda\right\} .
$$

If $\lambda \neq 0$ then $L_{\lambda}$ is smooth and is of dimension $n-1$. It is clear but must be noted that $L_{\lambda}$ and $L_{\lambda^{\prime}}$ are disjoint for $\lambda \neq \lambda^{\prime}$, and each point $p=\left(p_{1}, \ldots, p_{n}\right) \in \mathbb{C}^{n}$ is in a unique $L_{\lambda}$ where $\lambda=p_{1}^{2}+\cdots+p_{n}^{2}$. Hence the leaves $\left\{L_{\lambda}\right\}$ foliate $\mathbb{C}^{n}$, with a singularity which we describe below. 
The tangent space of $\mathbb{C}^{n}$ at $p$ is generated by the vectors

$$
\begin{gathered}
\overrightarrow{e_{1}}(p):=\left.\frac{\partial}{\partial X_{1}}\right|_{p}=\left(\begin{array}{c}
1 \\
\vdots \\
0
\end{array}\right) \\
\vdots \\
\overrightarrow{e_{n}}(p):=\left.\frac{\partial}{\partial X_{n}}\right|_{p}=\left(\begin{array}{c}
0 \\
\vdots \\
1
\end{array}\right) .
\end{gathered}
$$

The Jacobian of the defining equation for $L_{\lambda}$ at some $p \in L_{\lambda}$ is

$$
J(p)=\left(2 p_{1}, \ldots, 2 p_{n}\right) .
$$

The only singular set of the foliation is then the origin which lies in $L_{0}$. A vector $\vec{v} \in T_{p} \mathbb{C}^{n}$ is in $T_{p} L_{\lambda}$ if and only if

$$
J(p) \vec{v}=0,
$$

where $\vec{v}$ is considered as a column vector and the product is the usual matrix product. It follows then that the tangent space of $L_{\lambda}$ is generated by the vectors

$$
\overrightarrow{E_{i j}}(p):=2 p_{j} \overrightarrow{e_{i}}(p)-2 p_{i} \overrightarrow{e_{j}}(p), 1 \leq i<j \leq n
$$

which can be rewritten as

$$
\left.\vec{E}_{i j}(p)=\left(\begin{array}{c}
0 \\
\vdots \\
-2 p_{i} \\
\cdots \\
2 p_{j} \\
\vdots \\
0
\end{array}\right) \quad \begin{array}{l} 
\\
\vdots \\
\end{array}\right)
$$

for $1 \leq i<j \leq n$. Clearly $J(p) \vec{E}_{i j}=0$ and any other vector in the kernel of $J(p)$ is generated by these syzygies. The rank of the vector subspace of $T_{p} \mathbb{C}^{n}$ generated by these sections is $n-1$ if $p \neq 0$; for example if $p_{1} \neq 0$ then $\overrightarrow{E_{12}}(p), \overrightarrow{E_{13}}(p), \ldots, \overrightarrow{E_{1 n}}(p)$ are linearly independent and

$$
\overrightarrow{E_{i j}}(p)=\frac{p_{i}}{p_{1}} \overrightarrow{E_{i 1}}(p)-\frac{p_{j}}{p_{1}} \overrightarrow{E_{j 1}}(p), \text { for } 1<i<j \leq n .
$$


On the other hand $\overrightarrow{E_{i j}}(\underline{\mathbf{o}})=0$ for all $1 \leq i<j \leq n$. The $\overrightarrow{E_{i j}}$ 's thus generate an integrable sheaf $\xi$ whose leaves are the $L_{\lambda}$ 's. The rank of the vector space $T_{p}(\xi)$ is $n-1$ if $p \neq \underline{\mathbf{o}}$ and is 0 if $p=\underline{\mathbf{o}}$. We have shown that $\xi$ is of type $\operatorname{Fol}(n, n-1,0 ; 0)$.

The study of $\operatorname{Fol}(n, n-1,0 ; 0)$ is not exciting in its own right but we had a chance to develop our notation and perspective which are needed for the general case, without cluttering the geometric arguments below.

\section{2. $\operatorname{Fol}(n, n-2,1 ; 0)$.}

In the preceding example we dealt with a single equation defining the foliation. In this section we will deal with two equations preparing the way for the general case.

For each $\lambda=\left(\lambda_{1}, \lambda_{2}\right) \in \mathbb{C}^{2}$ define the leaf

$$
L_{\lambda}=\left\{\left(X_{1}, \ldots, X_{n}\right) \in \mathbb{C}^{n} \mid X_{1}=\lambda_{1}, X_{2}^{2}+\cdots+X_{n}^{2}=\lambda_{2}\right\} .
$$

Each $L_{\lambda}$ is smooth if $\lambda_{2} \neq 0$, and is of dimension $n-2$ in that case. For each $\lambda_{1} \in \mathbb{C}$ the leaf $L_{\left(\lambda_{1}, 0\right)}$ has an isolated singularity at the point $p=$ $\left(\lambda_{1}, 0, \ldots, 0\right) \in \mathbb{C}^{n}$. Thus the leaves $\left\{L_{\lambda}\right\}$ foliate $\mathbb{C}^{n}$ with a singularity along the first coordinate. If we denote the resulting foliation by $\xi$ then we claim that the dimension of the vector space $T_{\left(\lambda_{1}, 0, \ldots, 0\right)}(\xi)$ is zero for every $\lambda_{1} \in \mathbb{C}$.

The Jacobian of the defining equations for $L_{\lambda}$ is

$$
J(p)=\left(\begin{array}{cccc}
1 & 0 & \cdots & 0 \\
0 & 2 p_{2} & \cdots & 2 p_{n}
\end{array}\right) .
$$

A vector $\vec{v} \in T_{p} \mathbb{C}^{n}$ is in $T_{p} L_{\lambda}$ if and only if

$$
J(p) \vec{v}=\left(\begin{array}{l}
0 \\
0
\end{array}\right) .
$$

The vector space $T_{p} L_{\lambda}$ is generated by the vectors

$$
\overrightarrow{E_{i j}}(p) \text {, for } 2 \leq i<j \leq n \text {. }
$$

(Recall the Equations (20) and (21).) These sections generate a vector space of dimension $n-2$ if $p \neq\left(p_{1}, 0, \ldots, 0\right)$. If $p=\left(p_{1}, 0, \ldots, 0\right)$ then they generate the zero subspace. This puts $\xi$ in $\operatorname{Fol}(n, n-2,1 ; 0)$.

\section{3. $\operatorname{Fol}(n, k, r ; s)$ where $s=r+k+1-n \geq 0$.}

We are now ready for the general case. Take any set of integers $n, k, r$ with

$$
\begin{array}{r}
n>k>r \geq 0 \\
\text { and } r+k+1 \geq n .
\end{array}
$$


We then set

$$
s=r+k+1-n \text {. }
$$

By the assumption (24) we have $s \geq 0$. Moreover this together with the assumption (23) gives $r \geq s$. Hence our set of integers satisfy

$$
n>k>r \geq s=r+k+1-n \geq 0 .
$$

With the integers $n, k, r, s$ chosen as above define the leaf $L_{\lambda}$ for any $\lambda \in \mathbb{C}^{n-k}$

$$
\begin{aligned}
L_{\lambda}=\left\{\left(X_{1}, \ldots, X_{n}\right) \in \mathbb{C}^{n} \mid\right. & X_{1}, \ldots, X_{s} \text { are free } \\
& X_{s+1}=\lambda_{1} \\
& \vdots \\
& X_{r}=\lambda_{r}=\lambda_{n-k-1} \\
& \left.X_{r+1}^{2}+\cdots+X_{n}^{2}=\lambda_{n-k}\right\}
\end{aligned}
$$

where the implied claim in (28) follows from the assumption in (25). Note that if $r=s$ then by (25) $n-k=1$ and then we have only the Equation (29) in the definition of $L_{\lambda}$. The Equations (27)-(28) in that case do not appear.

With this understanding we describe the Jacobian for the defining equations of $L_{\lambda}$ as

$$
\begin{aligned}
& J(p)=\left(\begin{array}{ccccccccc}
0 & \cdots & 0 & 1 & \cdots & 0 & 0 & \cdots & 0 \\
\vdots & & \vdots & \vdots & \ddots & \vdots & & \vdots & \\
0 & \cdots & 0 & 0 & \cdots & 1 & 0 & \cdots & 0 \\
0 & \cdots & 0 & 0 & \cdots & 0 & 2 p_{r+1} & \cdots & 2 p_{n}
\end{array}\right)_{(n-k) \times n} \\
& \underbrace{\sim}_{s} \underbrace{\sim}_{r-s} \underbrace{r}_{n-r}
\end{aligned}
$$

The rank of $J(p)$ is $n-k$ if $p \neq\left(p_{1}, \ldots, p_{r}, 0, \ldots, 0\right)$, (note that $r-s=n-k-1$ by (25)). If $\lambda_{n-k} \neq 0$ then no point of the form $p=\left(p_{1}, \ldots, p_{r}, 0, \ldots, 0\right)$ is in $L_{\lambda}$ and the rank of $J(p)$ is $n-k$. The dimension of $L_{\lambda}$ is $k$.

If $\lambda_{n-k}=0$ then $L_{\lambda}$ is singular and the singularity set is defined by the equations $X_{r+1}=0, \ldots, X_{n}=0$ in addition to the Equations (27)-(28). This gives $(n-r)+(r-s)=n-s$ linear equations in $\mathbb{C}^{n}$ defining the singular set of $L_{\lambda}$, for each $\lambda=\left(\lambda_{1}, \ldots, \lambda_{n-k-1}, 0\right) \in \mathbb{C}^{n-k}$. Thus the dimension of the singularity of $L_{\lambda}$ is $s$, and these singularities are parametrized by $\mathbb{C}^{n-k-1}$, giving us a total of $s+(n-k-1)=(r+k+1-n)+(n-k-1)=r$ dimensions for the singularity of the foliation defined by the leaves $\left\{L_{\lambda}\right\}$. 
Denoting this foliation by $\xi$ we claim that the dimension of $T_{p}(\xi)$ is $s$ for $p$ on the singular locus of the foliation.

Going back to Equation (30) describing the Jacobian we see that the kernel of $J(p)$, and hence the vector subspace $T_{p}(\xi)$, is generated by the vectors

$$
\overrightarrow{e_{1}}(p), \ldots, \overrightarrow{e_{s}}(p) \text {, and } \overrightarrow{E_{i j}}(p) \text { for } r+1 \leq i<j \leq n
$$

for $p \neq\left(p_{1}, \ldots, p_{r}, 0, \ldots, 0\right)$, where we use the notation of Equations (18) and (20). The dimension of the vector space generated by these vectors is $s+(n-r-1)=(r+k+1-n)+(n-r-1)=k$ as expected since this is the dimension of each leaf at a smooth point. But if $p=\left(p_{1}, \ldots, p_{r}, 0, \ldots, 0\right)$ then each of the $\vec{E}_{i j}(p)$ 's in (31) vanish, leaving us with $\operatorname{dim} T_{p}(\xi)=s$. This then shows that $\xi$ is of type $\operatorname{Fol}(n, k, r ; s)$, where $s=r+k+1-n \geq 0$.

The notation of this example is so chosen that the relevant sets, such as $Z, Z_{\text {front }}, Z_{\text {back }}$ and $D$ can be described precisely by the Equations (6), (7), (8) and (9) respectively. The restriction of the foliation $\xi$ to the disk $D$ is a foliation $\eta$ of type $\operatorname{Fol}(n-s, k-s, r-s ; 0)$. It can now be shown that $\xi$ is obtained from $\eta$ by a first construction of type $s$, as in Section 2.3, i.e. $\xi=\eta_{s}$.

\subsection{Split Foliations.}

A split foliation can roughly be described as a parametrized foliation. (for the precise definition refer to the end of Section 2.4). The foliation $\xi$ described as in Example 4.2 is split. In fact each hyperplane $X_{1}=\lambda_{1}$ is foliated by leaves given by the equations $X_{2}^{2}+\cdots+X_{n}^{2}=\lambda_{2}$. This describes a foliation germ $\eta$ on $\mathbb{C}^{n-1}$ with leaves of rank $n-2$ and with an isolated singularity at the origin. Then $\eta$ is a germ in $\operatorname{Fol}(n-1, n-2,0 ; 0)$, as described in Example 4.1. It is now easy to see that $\xi$ is of the form $\ell_{1}(\eta)$, and hence is split.

The foliation $\eta$ on $D$, which is described at the end of Example 4.3, is of the form $\operatorname{Fol}(n-s, k-s, r-s ; 0)$. We claim that it is split. Each linear subspace $X_{s+1}=\lambda_{1}, \ldots, X_{r}=\lambda_{n-k-1}$ of $D$ is foliated by $\eta_{s}$ with a single equation $X_{n-k}^{2}+\cdots+X_{n}^{2}=\lambda_{n-k}$. Each such linear subspace is cut by $n-k-1$ equations, so is of dimension $(n-s)-(n-k-1)=n-r$, where we put $s=r+k+1-n$ as in (26). The restriction of $\eta_{s}$ to each such linear subspace foliates it with a single equation as described in Example 4.1. If we denote the restriction of $\eta_{s}$ to such a hyperplane by $\alpha$, then $\alpha$ is of type $\operatorname{Fol}(n-r, n-r-1,0 ; 0)$. The geometric set up suggests and in fact it can be shown that the $\eta_{s}$ in $\operatorname{Fol}(n-s, k-s, r-s ; 0)$ is obtained from the $\alpha$ in Fol $(n-r, n-r-1,0 ; 0)$ by a second construction of type $r-s$ as described in Section 2.4. i.e. $\eta_{s}=\ell_{r-s}(\alpha)$, and hence $\eta_{s}$ is split. To check that the 
relevant algebra also holds note that

$$
\begin{aligned}
\ell_{r-s}: \operatorname{Fol}(n-r, n-r-1,0 ; 0) \rightarrow & \operatorname{Fol}((n-r)+(r-s), n-r-1,(r-s) ; 0) \\
\| & \operatorname{Fol}(n-s, k-s, r-s ; 0)
\end{aligned}
$$

by putting $s=r+k+1-n$ as in (25).

\section{Closing Remarks.}

The examination of the structure of foliation singularities in the holomorphic category is motivated by the Rationality Conjecture of Baum and Bott in [2]. In the literature this conjecture is proved for a restricted class of foliations and a very restricted class of polynomials, the general case remaining still wide open. In this direction we hope that a close analysis of the singular set should prove helpful in understanding the behaviour of residues around the singularity.

The reader will no doubt notice that even though we dealt heavily with the geometry of the singularity, the problem is also related to the problem of extending coherent sheaves across a singularity. The algebraic behaviour of the sheaf is strongly tied with the geometry of the space.

At this point it is tempting, in the light of the examples discussed in Section 4 , to conjecture that any foliation $\xi$ of type $\operatorname{Fol}(n, k, r ; 0)$ with $n>$ $k>r$ and $n>k+r$ is split of the form $\ell_{r}(\alpha)$ for some $\alpha$ in $\operatorname{Fol}(n-r, k, 0 ; 0)$. This conjecture is meant to provoke further research rather than a call for fame, or the lack of it as the case might prove...

\section{References}

[1] P. Baum, Structure of foliation singularities, Advances in Math., 15 (1975), 361374.

[2] P. Baum and R. Bott, Singularities of holomorphic foliations, J. of Differential Geometry, 7 (1972), 279-342.

[3] D. Cerveau, Distributions involutives singulières, Ann. Inst. Fourier, Grenoble, 29 (1979), 261-294.

[4] J. Kwiecinski, private communications.

[5] A.T. Lundell, A short proof of the Frobenius theorem, Proc. Amer. Math. Soc., 116 (1992), 1131-1133.

[6] S. Sertöz, Residues of singular holomorphic foliations, Compositio Math., 70 (1989), 227-243. 
[7] F.W. Warner, Foundations of Differentiable Manifolds and Lie Groups, SpringerVerlag, 1983.

Received April 28, 1997.

BILKENT UNIVERSITY

06533 BILKENT

ANKARA, TURKEY

E-mail address: sertoz@fen.bilkent.edu.tr 